\title{
Production of Rubusoside Derivatives by Transgalactosylation of Various $\beta$-Galactosidases
}

\author{
Sumio Kitahata, Hiroshi IshiKawa, * Takeshi Miyata** \\ and Osamu TANAKA** \\ Osaka Municipal Technical Research Institute, 1-6-50, Morinomiya, \\ Jyoto-ku, Osaka 536 , Japan \\ * Hokkaido Sugar Co., Ltd., 2-1, Jinbocho, Kanda, Chiyoda-ku, \\ Tokyo 10I, Japan \\ **Institute of Pharmaceutical Sciences, Hiroshima University \\ School of Medicine, Kasumi, Minami-ku, \\ Hiroshima 734, Japan \\ Received May 12, 1989
}

\begin{abstract}
Rubusoside derivatives by transgalactosylation of various $\beta$-galactosidases were isolated and their structures were analyzed. Escherichia coli $\beta$-galactosidase produced mainly 13-O- $\beta$-D-glucosyl-19-O[ $\beta$-D-galactosyl-( $1 \rightarrow 6)-\beta$-D-glucosyl]-steviol (RGal-2). Bacillus circulans $\beta$-galactosidase produced mainly 13-O- $\beta$-D-glucosyl-19- $O$ - $[\beta$-D-galactosyl-(1 $\rightarrow 4)-\beta$-D-glucosyl]-steviol (RGal-1a) in the early

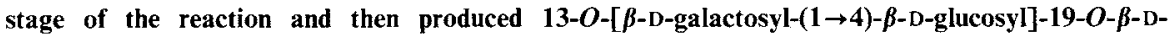
glucosyl-steviol (RGal-1b). With decreasing the amount of these products (RGal-1a and RGal-1b), RGal-2 was produced.
\end{abstract}

Rubusoside ( $\beta$-D-glucosyl ester of 13-O- $\beta$-Dglucosyl-steviol) is isolated from the leaves of Rubus suavissimus S. Lee (Rosaceae) collected in Kwangchow, China. ${ }^{1,2)}$ Rubusoside is about 110 times as sweet as sucrose at the concentration of $0.025 \%$. Rubusoside, however, has a slightly bitter taste.

Darise et al. ${ }^{3)}$ synthesized various rubusoside derivatives by transglycosylation by cyclomaltodextrin glucanotransferase (CGTase) and studied the relationship between the chemical structures and their tastes. Evaluation of the sweetness and quality of the taste of these derivatives disclosed that sweetness and its quality of taste were greatly improved by the transglycosylation of the glucosyl residue at the 13-hydroxyl group, while a change of sweetness and quality of taste for the worse was observed with transglycosylation to the glucosyl residue at the 19-carboxyl group.

It is already known that glucose is an efficient acceptor for the intermolecular transglycosylation of CGT-ase, but galactose is not an efficient acceptor. ${ }^{4)}$ Recently, we found that the $\beta$-galactosidase from Bacillus circulans transferred a galactosyl residue preferentially to the C4-hydroxyl group of the glucosyl residue at the 19-carboxyl group of rubusoside under certain conditions. ${ }^{5}$ Using this transgalactosylation product as an acceptor of CGT-ase action, rubusoside derivatives which are transglycosylated preferentially on the glucosyl residue at the 13-hydroxyl group of rubusoside will be produced. This paper deals with the structural elucidation of transgalactosylated derivatives of rubusoside by $\beta$ galactosidases from various origins.

\section{Materials and Methods}

Materials. Rubusoside was isolated from leaves of a Rubus suavissimus (Rosaceae) collected in Kwangchow, China by the method of Tanaka et al." Lactose was purchased from Wako Pure Chemical Industries. $\beta$ Galactosidases from $B$. circulans, Aspergillus oryzae, Penicillium multicolor, and Kluyveromyces lactis were supplied by Daiwa Kasei K.K., Yakult Honsha Co., Ltd., K·I Chemical Industry Co., Ltd., Godo Shusei Co., Ltd., respectively. $\beta$-Galactosidase from Escherichia coli was 
purchased from Boehringer Mannheim GMBH Biochemica.

Assay of $\beta$-galactosidase activity. $\beta$-Galactosidase activity was assayed by the spectrophotometric measurement of the release of $p$-nitrophenol from PNP- $\beta$-galactoside. The reaction mixture containing $200 \mu \mathrm{l}$ of $10 \mathrm{mM}$ PNP- $\beta$ galactoside, $200 \mu \mathrm{l}$ of $40 \mathrm{~mm} \mathrm{pH} 6.0$ acetate buffer, and $50 \mu \mathrm{l}$ of enzyme solution was incubated at $40^{\circ} \mathrm{C}$. After $10 \mathrm{~min}$, the reaction was stopped by adding $500 \mu \mathrm{l}$ of $200 \mathrm{~mm}$ sodium carbonate. One unit of $\beta$-galactosidase activity is defined as the amount releasing $1 \mu \mathrm{mol}$ of $p$ nitrophenol per min.

High performance liquid chromatography. High performance liquid chromatography (HPLC) for the measurement of the transgalactosylation products was done under the following conditions; column, TSK gel ODS-120T $(4.6 \times 250 \mathrm{~mm})$; detector, Shimadzu SPD-6A $(213 \mathrm{~nm})$; solvent, acetonitrile-water $(25 \sim 30: 75 \sim 70, v / v)$ and flow rate, $0.5 \mathrm{ml} / \mathrm{min}$. Conditions for preparative HPLC were as follows; column, TSK gel ODS-120T $(21.5 \times 300 \mathrm{~mm})$; detector, Shodex RE-11 refractometer; solvent, acetonitrile-water $(30: 70, \mathrm{v} / \mathrm{v})$ and flow rate, $6 \mathrm{ml} / \mathrm{min}$.

Thin layer chromatography. Thin layer chromatography (TLC) was done on a plate of Kiesel gel 60 TLC plate (Merck Co., Ltd.) with a solvent system of chloroformmethanol-water $(10: 5: 1$ or $6: 4: 1, \mathrm{v} / \mathrm{v})$. The sugars on the TLC plate were revealed by heating at $110 \sim 120^{\circ} \mathrm{C}$ after spraying with sulfuric acid-methanol.

Measurement of nuclear magnetic resonance (NMR) spectra. NMR spectra were recorded with a JEOL GX-400 spectrometer at $28^{\circ} \mathrm{C}$ for pyridine- $d_{5}$ solutions at $400 \mathrm{MHz}$ for protons and at $100 \mathrm{MHz}$ for carbon-13 with tetramethylsilane as an internal standard.

Optical rotation. $[\alpha]_{\mathrm{D}}$ was measured on a Union automatic digital polarimeter.

Selective cleavage of ester type glycoside linkages. ${ }^{6)} \mathrm{A}$ mixture of a glycoside ( $5 \mathrm{mg}$ ), anhydrous Lil ( $5 \mathrm{mg}$ ), and anhydrous 2,6 -lutidine $(3 \mathrm{ml})$ in anhydrous methanol $(3 \mathrm{ml})$ was refluxed for $8 \mathrm{hr}$. The mixture was diluted with $50 \%$ methanol and then deionized with Amberlite MB-3. In TLC, the $R f$ value of the resulting 13-O-glycosyl-steviol decreases in the order of 13-O-mono-, di-, and triglycosides, readily distinguishing these compounds from each other.

\section{Results}

Transgalactosylation of rubusoside by $B$. circulans $\beta$-galactosidase

Reaction mixtures containing $1.1 \mathrm{M}$ lactose

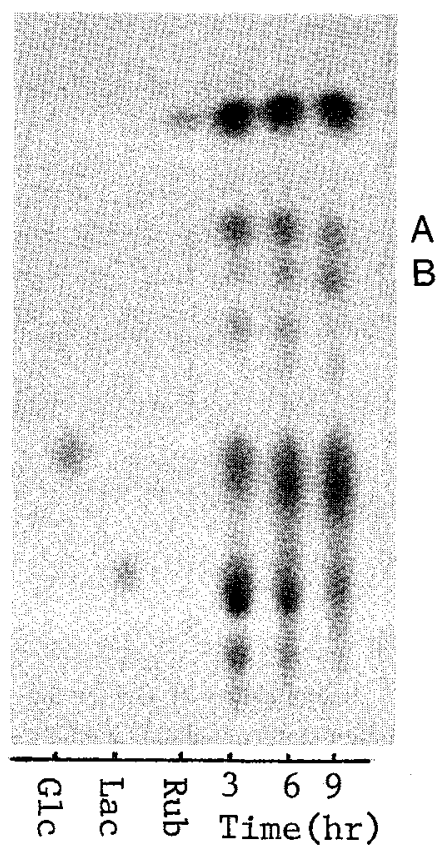

Fig. 1. Thin Layer Chromatogram of $\beta$-Galactosidase Action Products on the Mixture of Lactose and $\mathrm{Ru}$ busoside.

Glc, glucose; Lac, lactose; Rub, rubusoside; A and B, transfer products to rubusoside.

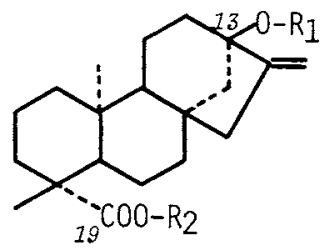

\begin{tabular}{lll} 
Compound & \multicolumn{1}{c}{$\mathrm{R}_{1}$} & \multicolumn{1}{c}{$\mathrm{R}_{2}$} \\
Rubusoside & $\beta-G l c$ & $\beta-G I c$ \\
RGa1-1a & $\beta-G 1 c$ & $\beta-G 1 c^{4}-{ }^{1} \beta-G a 1$ \\
RGal-1b & $\beta-G 1 c^{4}-{ }^{1} \beta-G a 1$ & $\beta-G 1 c$ \\
RGa1-2 & $\beta-G 1 c$ & $\beta-G 1 c^{6}-{ }^{1} \beta-G a I$ \\
RGal-3 & $\beta-G 1 c^{6}-{ }^{1} \beta-G a 1$ & $\beta-G l c$
\end{tabular}

as donor, $0.55 \mathrm{M}$ rubusoside as acceptor and $\beta$ galactosidase (150 units) in a total volume of $200 \mu \mathrm{l}$ of $50 \mathrm{~mm}$ acetate buffer (pH 6.0) were incubated at $40^{\circ} \mathrm{C}$. After $0.5,1.0$, and $3.0 \mathrm{hr}$, $50 \mu \mathrm{l}$ samples of the reaction mixture were removed to examine by TLC and HPLC. As shown in Fig. 1, new spots (A and B) had appeared between the $R f$ value of rubusoside 
and that of glucose. These new spots seemed to be transgalactosylated derivatives of rubusoside, judging from their $R f$ values. By HPLC analysis, three peaks, RGal-2, RGal-3, and RGal-1, were detected in this order except for rubusoside. The spots $\mathrm{A}$ and $\mathrm{B}$ on TLC plate correspond to RGal-1 and the mixture of RGal-2 and RGal-3, respectively.

\section{Isolation of transgalactosylated products}

Fourteen $\mathrm{ml}$ of the reaction mixture containing $5.0 \mathrm{~g}$ of rubusoside, $5.6 \mathrm{~g}$ of lactose, and the enzyme solution (5040 units) was incubated at $40^{\circ} \mathrm{C}$. After $7.0 \mathrm{hr}$, the reaction mixture was heated at $100^{\circ} \mathrm{C}$ for $10 \mathrm{~min}$ to inactivate the enzyme and centrifuged to remove the insoluble materials. The supernatant solution was put on a column $(30 \times 210 \mathrm{~mm})$ of DIAION HP-20 (Nippon Rensui Co.). After eluting with water to remove mono- and oligosaccharides, a mixture of the galactosylated products and the starting material (rubusoside) was eluted with $70 \%$ methanol. This mixture was chromatographed on silica gel by linear gradient elution with chloroformmethanol-water $(30: 10: 1-15: 7: 1-10: 5: 1$, homogeneous) to give rubusoside $(3.2 \mathrm{~g})$ and a mixture of the products, the latter of which were further separated by preparative HPLC, yielding RGal-2 (775 mg), RGal-3 (30 mg), and RGal-1 (10 mg).

\section{Structural elucidation of transgalactosylated products}

RGal- 2 was obtained as a white powder, $[\alpha]_{D}$ $-40.2^{\circ}(c=1.01$, methanol) Anal. Found: C, $54.55 ; \mathrm{H}, 7.56 \%$. Calcd. for $\mathrm{C}_{38} \mathrm{H}_{60} \mathrm{O}_{18} \cdot 2 \mathrm{H}_{2} \mathrm{O}$ : $\mathrm{C}, 54.27 ; \mathrm{H}, 7.67 \%$. In the NMR spectra, RGal-2 had three anomeric proton signals at $\delta$ $6.05(1 \mathrm{H}, \mathrm{d}, J=7.3 \mathrm{~Hz}, 19-O-\beta-\mathrm{Glc}), 5.14(1 \mathrm{H}$ $\mathrm{d}, J=7.7 \mathrm{~Hz}), 4.96(1 \mathrm{H} \mathrm{d}, J=7.7 \mathrm{~Hz}, \beta$-Gal $)$, and three anomeric carbon signals at $\delta 105.8$ $\left(\beta\right.$-Gal), $99.7(13-O-\beta-\mathrm{Glc})^{7)}$, and 95.7 (19COO- $\beta$-Glc). ${ }^{7)}$ This indicated that $\mathrm{RGal}-2$ must be a mono- $\beta$-D-galactosyl-rubusoside. RGal-2 had two carbon signals at $\delta 62.2$ and 63.0 due to non-substituted C- 6 of glucosyl and galactosyl groups, while a carbon signal at
69.6 can be assigned as C-6 of a 6-linked glucosyl group. This showed that the 6-hydroxyl group of one of the two $\beta$-D-glucosyl units was galactosylated. The allocation of the galactosyl group at 19-COO-glucosyl unit was established by the result of the selective cleavage of an ester type glycosyl linkage; TLC of the product showed the formation of $13-O-\beta$ D-glucosyl-steviol (steviolmonoside). It follows that RGal-2 can be formulated as $13-O-\beta$-Dglucosyl-19- $O$ - $[\beta$-D-galactosyl- $(1 \rightarrow 6)-\beta$-Dglucosyl]-steviol.

RGal-3 was obtained as a white powder, $[\alpha]_{\mathrm{D}}$ $-40.9^{\circ}(c=0.5$, methanol). Anal. Found: $\mathrm{C}$, $54.29 ; \mathrm{H}, 7.53 \%$. Calcd. for $\mathrm{C}_{38} \mathrm{H}_{60} \mathrm{O}_{18} \cdot 2 \mathrm{H}_{2} \mathrm{O}$ : $\mathrm{C}, 54.27 ; \mathrm{H}, 7.67 \%$. Three anomeric proton signals at $\delta 6.16(1 \mathrm{H}, \mathrm{d}, J=8.1 \mathrm{~Hz}, 19-C O O-\beta$ Glc) and $5.06(2 \mathrm{H} \mathrm{d}, J=7.3 \mathrm{~Hz}, \beta$-Gal $)$ as well as three anomeric carbon signals at $\delta 95.9$ (19COO- $\beta$-Glc), 99.6 (13-O- $\beta$-Glc), and 105.1 ( $\beta$ Gal), indicated that RGal-3 must be also a mono- $\beta$-galactosylated product. As in the case of RGal-2, RGal-3 had a carbon signal at $\delta$ 69.7 assignable to $C-6$ of a 6-linked glucosyl group together with two non-substituted C-6 signals of glycosyl and galactosyl groups at $\delta$ 62.2 and 62.3 .

On the selective cleavage of an ester type glycosyl linkage in the same way as RGal-2, RGal-3 afforded a glycoside (RGal-3-G). The TLC- $R f$ value of RGal-3-G is evidently lower than steviolmonoside, being similar to that of 13-O- $\beta$-sophorosyl-steviol (steviolbioside), indicating that $\mathrm{RGal}-3-\mathrm{G}$ can be assigned to 13-O-galactosyl-D-glucosyl-steviol. Based on these results, RGal-3 can be formulated as 13$O$-[ $\beta$-D-galactosyl- $(1 \rightarrow 6)-\beta$-D-glucosyl]- 19- $O$ $\beta$-glucosyl-steviol.

The ${ }^{13} \mathrm{C}-\mathrm{NMR}$ spectrum showed that RGal1 mainly consists of another mono- $\beta$ galactosylated product (RGal-1a) with a small amount of an isomeric product (RGal-1b). Separation of the isomers from each other has not been done. RGal-1 was selectively cleaved at an ester type glycosyl linkage in the same way as RGal-2 and the TLC of the products revealed the formation of steviolmonoside along with a small amount of a by-product 
Table 1. Effects of Substrate Concentration on the Production of RGal-1, RGal-2, AND RGal-3 BY $B$. circulans $\beta$-GALACTOSIDASE

\begin{tabular}{|c|c|c|c|c|c|c|c|}
\hline \multirow{2}{*}{$\begin{array}{c}\text { Lactose (M) } \\
\text { Rubusoside (M) }\end{array}$} & \multirow{2}{*}{ Product } & \multicolumn{6}{|c|}{ Reaction time (hr) } \\
\hline & & 1.5 & 3.0 & 6.0 & 10 & 12 & 36 \\
\hline 0.28 & RGal-1 & $16 \%$ & $6.8 \%$ & $1.6 \%$ & $1.3 \%$ & $0.7 \%$ & $0.3 \%{ }^{a}$ \\
\hline \multirow[t]{2}{*}{0.14} & RGal-2 & 6.9 & 11 & 5.8 & 1.9 & 1.1 & 0.5 \\
\hline & RGal-3 & 0.6 & 1.1 & 1.7 & 1.6 & 1.3 & 1.0 \\
\hline 0.56 & RGal-1 & 17 & 11 & 5.2 & 3.0 & 1.7 & 0.4 \\
\hline \multirow[t]{2}{*}{0.28} & RGal-2 & 5.9 & 10 & 11 & 9.0 & 2.5 & 1.8 \\
\hline & RGal-3 & 0.5 & 1.0 & 1.7 & 1.7 & 1.8 & 1.4 \\
\hline 0.83 & RGal-1 & 19 & 14 & 9.0 & 5.6 & 3.8 & 2.7 \\
\hline \multirow[t]{2}{*}{0.42} & RGal-2 & 4.8 & 8.9 & 11 & 12 & 7.3 & 4.2 \\
\hline & RGal-3 & 0.4 & 1.1 & 1.0 & 1.7 & 1.6 & 2.0 \\
\hline 1.1 & RGal-I & 19 & 17 & 13 & 11 & 5.4 & 4.3 \\
\hline \multirow[t]{2}{*}{0.55} & RGal-2 & 4.4 & 7.6 & 10 & 12 & 9.2 & 8.6 \\
\hline & RGal-3 & 0.4 & 0.7 & 1.2 & 1.4 & 2.2 & 2.6 \\
\hline
\end{tabular}

a [Amount of transfer product (M)/Amount of rubusoside used $(\mathrm{M})] \times 100$.

which showed the similar TLC-Rf value to that of steviolbioside, being recognized as a 13-O-galactosyl-glucosyl-steviol. These results suggested that RGal-1a is formulated as 13$O$ - $\beta$-D-glucosyl-19- $O$ - $(\beta$-D-galactosyl- $\beta$-Dglucosyl)-steviol. This major product (RGalla) was obtained without contamination with $\mathrm{RGal}-1 \mathrm{~b}$ under the other reaction conditions (see below) and the formulation was finally established for th pure sample.

\section{Effects of substrate concentration and reaction time on the formation of RGal-1, RGal-2, and RGal-3}

For the purpose of investigation of the effects of lactose and rubusoside concentration on RGal-1, RGal-2, and RGal-3 formation, $\beta$-galactosidase from $B$. circulans $(1880$ units/lactose, g) was incubated with various concentrations of lactose and rubusoside at $40^{\circ} \mathrm{C}$. After $1.5,3.0,6.0,10,12$, and $36 \mathrm{hr}$, the amounts of RGal-1, RGal-2, and RGal-3 produced in the reaction mixture were measured by HPLC. As shown in Table I, the amounts of RGal-1, RGal-2, and RGal-3 were slightly increased with the increase of substrate concentration. The RGal-1 was formed significantly in the early stage (within $1.5 \mathrm{hr}$ ) and
Table II. Course of The Production of RGal-1a, RGal-1b, RGal-2, AND RGal-3 BY $B$. circulans $\beta$-GALACTOSIDASE

\begin{tabular}{lcccc}
\hline & \multicolumn{5}{c}{ Reaction time (min) } \\
\cline { 2 - 5 } Product & 20 & 50 & 70 & 160 \\
\hline RGal-1a & $13.3 \%$ & $16.7 \%$ & $15.0 \%$ & $9.8 \%{ }^{a}$ \\
RGal-1b & 2.5 & 2.6 & 4.6 & 7.3 \\
RGal-2 & 1.6 & 2.6 & 3.8 & 6.2 \\
RGal-3 & 0.4 & 0.3 & 0.7 & 0.6 \\
\hline
\end{tabular}

Reaction conditions: lactose, $0.28 \mathrm{M}$; rubusoside, $0.14 \mathrm{M}$.

a [Amount of transfer product (M)/Amount of rubusoside used $(\mathrm{M})] \times 100$.

then decreased rapidly, followed by the formation of RGal-2. A small amount of RGal-3 was formed at last.

Based on this evidence, the course of the production of RGal-1 was investigated for a shorter period. A mixture $(40 \mathrm{ml})$ of $0.28 \mathrm{M}$ lactose and $0.14 \mathrm{M}$ rubusoside was incubated with $\beta$-galactosidase from $B$. circulans $(7500$ units) at $40^{\circ} \mathrm{C}$. After $20,50,70$, and $160 \mathrm{~min}$, $10 \mathrm{ml}$ samples were removed for column chromatography on DIAION HP-20 and then on silica gel to give RGal-1-fractions in yields of 15.8 (20 $\mathrm{min}), 19.3$ (50 $\mathrm{min}), 19.6$ (70 $\mathrm{min})$, and $17.1 \%$ (160 min) (Table II). The concentration 
of $\mathrm{RGal}-1$ reached a maximum after $70 \mathrm{~min}$. The ratio of RGal-1a and $R$ Gal-1b in RGal-1 at each reaction time was measured approximately by comparison of the strength of the anomeric proton signal due to $19-\mathrm{COO}-\beta$-Glc; RGal-1a $\delta 6.03(1 \mathrm{H} \mathrm{d}, J=7.7 \mathrm{~Hz})$ and RGallb $\delta 6.15(1 \mathrm{H} \mathrm{d}, J=8.06 \mathrm{~Hz})$. It was found that the RGal- 1 after 20 and 50 min reaction consisted of RGal-1a almost exclusively, while the contamination of RGal-1b was increased with reaction time. A pure specimen of RGal-1a was obtained by recrystallization of RGal-1fraction after $20 \mathrm{~min}$ from methanol $-\mathrm{H}_{2} \mathrm{O}$.

RGal-1a: colorless needles, mp $205 \sim 209^{\circ} \mathrm{C}$, $[\alpha]_{\mathrm{D}}-24.4\left(c=0.92, \mathrm{C}_{5} \mathrm{H}_{5} \mathrm{~N}\right)$. Anal. Found: C, $53.19 \% ; \mathrm{H}, 7.64 \%$. Calcd. for $\mathrm{C}_{38} \mathrm{H}_{60} \mathrm{O}_{18}$. $3 \mathrm{H}_{2} \mathrm{O}: \mathrm{C}, 53.13 \% ; \mathrm{H}, 7.75 \%$. The ${ }^{1} \mathrm{H}-\mathrm{NMR}$ spectrum of RGal-1a exhibited anomeric proton signals at $\delta 6.03(1 \mathrm{H} \mathrm{d}, J=7.7 \mathrm{~Hz})$ and $5.13(2 \mathrm{H} \mathrm{d}, J=7.7 \mathrm{~Hz})$. The ${ }^{13} \mathrm{C}-\mathrm{NMR}$ spectrum of RGal-1a had the following signals which are diagnostic for the structure elucidation: at $\delta 95.5$ (anomeric carbon of 19-COO$\beta$-Glc), 99.7 (anomeric carbon of $13-O-\beta$-Glc), 105.7 (anomeric carbon of $\beta$-Gal), 62.9, 62.0, and 61.4 (three unsubstituted C-6 of Gal and $2 \times \mathrm{Glc}$ ), and 80.9 (C-4 of 4-linked Glc): This indicated that one of the 4-hydroxyl groups of the two $\beta$-glucosyl units was $\beta$-galactosylated in RGal-1a. Based on these spectral data coupled with the results of the cleavage of the ester glycoside linkage for RGal-1 mentioned above, RGal-1a can be formulated as 13- $O-\beta$-D-glucosyl-19- $O$-[ $\beta$-D-galactosyl$(1 \rightarrow 4)$ - $\beta$-D-glucosyl]-steviol. The minor product RGal-1b in RGal-1 has not been purified. The inspection of the minor signals of the ${ }^{13} \mathrm{C}$ NMR spectrum of RGal-1 as well as the result of the cleavage of ester glycoside linkage for RGal-1 (see above) suggested that RGal-1b would be assignable to $13-O-[\beta$-D-galactosyl$(1 \rightarrow 4)-\beta$-D-glucosyl]-19- $O$ - $\beta$-D-glucosylsteviol.

\section{Comparison of transgalactosylated products by} $\beta$-galactosidases from various origins

$\beta$-Galactosidases from $B$. circulans, E. coli, Asp. oryzae, P. multicolor, and K. lactis (40
Table III. Transfer Products of Rubusoside By Various $\beta$-Galactosidases ${ }^{a}$

\begin{tabular}{lcccc}
\hline & B. circulans & E. coli & $\begin{array}{c}\text { Asp. } \\
\text { oryzae }\end{array}$ & P. multicolor \\
\hline RGal-1 & $13.6 \%$ & $2.3 \%$ & $1.1 \%$ & $1.7 \%$ \\
RGal-2 & 12.3 & 12.5 & 3.2 & 3.8 \\
RGal-3 & 0.9 & 0.5 & 2.2 & 2.3 \\
Unknown $^{c}$ & - & - & 0.8 & 1.0 \\
\hline
\end{tabular}

a Amount of transfer products after $15 \mathrm{hr}$ of reaction.

${ }^{b}$ [Amount of transfer product (M)/Amount of rubusoside used $(M)] \times 100$.

c Unknown, structure of the transfer product is not identified.

50 units) were incubated with a mixture of $1.1 \mathrm{M}$ lactose and $0.55 \mathrm{M}$ rubusoside $(400 \mu \mathrm{l})$ at $40^{\circ} \mathrm{C}$. After $1,2,3,5,7$, and $15 \mathrm{hr}, 10 \mu \mathrm{l}$ samples of the reaction mixture were removed to analyze by HPLC. The amount of each transfer product increased with reaction time. As shown in Table III, $\beta$-galactosidases from $B$. circulans and $E$. coli produced RGal-1, RGal2 , and RGal-3. On the other hand, $\beta$-galactosidases from Asp. oryzae and $P$. multicolor produced RGal-1, RGal-2, RGal-3 and an unidentified product. $K$. lactis $\beta$-galactosidase produced no transgalactosylated derivatives of rubusoside.

\section{Discussion}

$B$. circulans $\beta$-galactosidase transferred a galactosyl residue preferentially to the glucosyl residue at the 19-carboxyl group of rubusoside at the $\beta-1,4$-linkage in the early stage of reaction, and produced RGal-1a, and then produced RGal-1b. With decreasing the amount of RGal-1 (RGal-1a and RGal-1b), RGal-2 was produced. On the other hand, E. coli $\beta$-galactosidase transferred a galactosyl residue mainly to the glucosyl residue at the carboxyl group of rubusoside by $\beta-1-6-$ linkage and produced RGal-2. $\beta$-Galactosidases from $A s p$. oryzae and $P$. multicolor produced RGal-1, RGal-2, RGal-3 and an unidentified product, though the amount of transfer products was small.

The main transfer product in $E$. coli $\beta$ - 
galactosidase, RGal-2, has a free C4-hydroxyl group in the glucosyl residue at the 19-carboxyl group. Consequently, it was not a good acceptor of transglycosylation by CGT-ase for the preferentially synthesis of the transglycosylation products to the glucosyl residue at the 13-hydroxyl group of rubusoside. (This result will be published in our next paper.) The transfer product in the early stage of reaction by $B$. circulans $\beta$-galactosidase, RGal-1a, will be a good acceptor for this purpose, because of the C4-hydroxyl group in the glucosyl residue at the 19-carboxyl group being blocked by galactose.

Mizutani et al ${ }^{8)}$ synthesized rubusoside and stevioside derivatives chemically, in which the $\beta$-D-glucosyl ester group at the 19-carboxylic acid was replaced by a $\beta$-D-galactosyl group. Using these derivatives as acceptors of CGTase, selective elongation of the glucosyl residue to the 13-O-glucosyl moiety of steviol bisglycosides was done to improve sweetness and quality of taste. But this chemical method to synthesize these derivatives is impractical, because of using various organic solvents and metallic salts. On the other hand, an enzymic method using $\beta$-galactosidase from $B$. circulans is practical to synthesize sweeteners for foods, and this method will be also applicable to stevioside.

\section{References}

1) T. Tanaka, H. Kohda, O. Tanaka, F.-H. Chen, W. -H. Chou and J.-L. Leu, Agric. Biol. Chem., 45, 2165 (1981).

2) O. Tanaka, Kagaku to Seibutsu, 24, 318 (1986).

3) M. Darise, K. Mizutani, R. Kasai, O. Tanaka, S. Kitahata, S. Okada, S. Oagaw, F. Murakami and F. -H. Chen, Agric. Biol. Chem., 48, 2483 (1984).

4) S. Kitahata, S. Okada and T. Fukui, Agric. Biol. Chem., 42, 2369 (1978).

5) S. Kitahata, H. Ishikawa, T. Miyata and O. Tanaka, Abstracts of Papers, the Annual Meeting of the Agricultural Chemical Society of Japan, Niigata, April, 1989, p. 291

6) K. Ohtani, K. Mizutani, R. Kasai and O. Tanaka, Tetrahedron Lett., 25, 4537 (1984).

7) K. Yamasaki, H. Kohda, T. Kobayashi, R. Kasai and $\mathrm{O}$. Tanaka, Tetrahedron Lett., 1976, 1005.

8) K. Mizutani, T. Miyata, R. Kasai, O. Tanaka, S. Ogawa and S. Doi, Agric. Biol. Chem., 53, 395 (1989). 